\title{
EDITORIAL
}

\section{Pandemic influenza: using evidence on vaccines and antivirals for clinical decisions and policy making}

\author{
A. Granados*, C. Goodman ${ }^{\#}$ and L. Eklund
}

I nfluenza pandemics have occurred approximately every 10-50 yrs for at least several centuries, including three times in the 20th century. Influenza viruses can be highly contagious, spreading rapidly throughout regional and global populations. Furthermore, they can mutate rapidly and develop resistance to available treatments. Symptoms are generally of sudden onset, including fever, headache, cough, sore throat, aching muscles, severe weakness and respiratory symptoms of varying severity.

\section{THE ISSUE}

A new influenza pandemic is inevitable. The type A H5N1 avian influenza is a potential pandemic threat. This influenza virus is currently circulating in Asia and has appeared in other regions, including Europe, the Middle East and Africa. More than 150 million birds have died from the virus or have been killed in an attempt to limit its spread. It has been transmitted from birds to humans on a limited basis. The reported human fatality rate is $>50 \%$, based on at least 91 deaths among at least 169 confirmed cases reported to the World Health Organization (WHO) since December 2003.

Avian influenza has jumped from birds to humans on several occasions during the last century (Hong Kong 1997, H5N1; Hong Kong 1999, H9N2; the Netherlands 2003, H7N7; Hong Kong 2003, H5N1; and Cambodia, Iraq, Indonesia, Vietnam, Thailand and Turkey 2004-2006, H5N1). Genetic research reported in 2005 revealed that the 1918-1920 pandemic was caused by an H1N1 virus closely related to avian viruses, and probably originated from a bird-human transmission [1]. If a form of $\mathrm{H} 5 \mathrm{~N} 1$ emerges that is readily transmissible among humans and spreads rapidly, it will pose a great threat to global public health. The recent outbreaks of avian influenza have triggered extraordinary attention from the mass media and have heightened public concern, along with public health alerts and major financial commitments by national governments, international agencies and industry. Many of these actions are based on limited evidence accompanying great uncertainty of an evolving threat.

\footnotetext{
*Universitat de Barcelona, Institut Municipal d'Investigació Mèdica (IMIM), Respiratory and Environmental Health Research Unit, Barcelona, Spain. ${ }^{\#}$ The Lewin Group, Falls Church, VA, USA "Health Evidence Network, DCS, WHO Regional Office of Europe, Copenhagen, Denmark.

CORRESPONDENCE: A. Granados, Institut Municipal d'Investigació Mèdica, Respiratory and Environmental Health Research Unit, Doctor Aiguader 80, 08003 Barcelona, Spain. Fax: 34 932216448. E-mail: agranados@imim.es
}

Based in part on a recent synthesis report undertaken by the Health Evidence Network (HEN; www.euro.who.int/HEN), this editorial highlights relevant available evidence for chest physicians and general practitioners confronting scientific and public uncertainties of the next influenza pandemic. (HEN is an information service of the WHO Regional Office of Europe that uses evidence-based approaches to respond to concrete and explicit public health questions and policy concerns.)

\section{THE QUESTION}

In the context of a mounting epidemiological threat of uncertain but potentially enormous health, social and economic consequences, the question to WHO HEN in 2005 was "How effective would antiviral vaccination and antiviral drug prevention and treatment strategies be for reducing the impact of the next influenza pandemic?"

\section{SOURCES AND TYPE OF EVIDENCE}

The HEN report is based on a comprehensive search of peerreviewed, published literature (including PubMed/MEDLINE, the Cochrane Databases, EMBASE and other key literature databases) and reports or guidance documents from agencies involved in public health response (e.g. WHO, the European Union Commission on Community Influenza Preparedness and Response, and US Centers for Disease Control and Prevention (CDC)). The HEN document explicitly examines available systematic reviews with meta-analyses, prospective and retrospective observational studies, practice guidelines, narrative (non-systematic) reviews and grey literature.

\section{FINDINGS}

Direct evidence of the effectiveness of specific vaccine prevention and antiviral prevention and treatment strategies for reducing the health impacts of a pandemic is extremely limited. Many of the newer vaccines and antiviral drug interventions being suggested for use in a potential emerging pandemic have only been tested during the last few years in small clinical trials involving healthy patients and not in people actually exposed to influenza or in the context of an actual epidemic or pandemic. Vaccines are the most effective primary strategy available for preventing and lowering the impact of an influenza outbreak. A systematic review of randomised controlled trials (RCTs) of influenza prevention concludes that vaccination with both inactivated and liveattenuated vaccines is moderately effective in healthy adults and children $>6$ months old [2]. A recent systematic review of the available evidence of effectiveness of influenza vaccines in the elderly found more modest benefits than those generally 
cited by national and international agencies, particularly where the match between the vaccine strains and the circulating strains was poor or unknown [3].

Antiviral drugs for influenza currently include two main classes, each with two drugs: M2 ion channel inhibitors, i.e. amantadine and rimantadine, and neuraminidase inhibitors (NAIs), including oseltamivir (Tamiflu; Roche Laboratories, Nutley, NJ, USA) and zanamivir (Relenza; GlaxoSmithKline, Uxbridge, UK). Most influenza strains that cause epidemics and pandemics are variations of the influenza A (the more pathogenic for humans) or B viruses. Both drug classes have shown partial effectiveness for prevention and treatment of influenza A viruses. NAIs, but not M2 inhibitors, are also active against influenza B viruses. Very recently, there is sufficient persuasive evidence of growing resistance of seasonal influenza A viruses to M2 inhibitors to prompt the US CDC to issue recommendations against using these antivirals during the current influenza season [4].

These two classes of antiviral agents have not been compared in an RCT. Prevention using either NAI reduces the risk of contracting laboratory-confirmed influenza by $\sim 70-90 \%$ compared with placebo. In previously healthy people who have contracted the influenza virus, antivirals can reduce the severity and duration of the infection, but only if taken within $36-48 \mathrm{~h}$ of the onset of illness; hence a rapid diagnosis is required. There are fewer RCTs pertaining to treatment in high-risk populations and little evidence regarding reduction of hospitalisations and mortality in all groups. Both M2 inhibitors can induce significant adverse gastrointestinal effects. Amantadine is more likely than rimantadine to induce certain central nervous system side-effects, including delirium and seizures, primarily in elderly people on higher doses $[5,6]$. NAIs generally have fewer side-effects than M2 inhibitors, although oseltamivir causes a somewhat higher rate of nausea and vomiting.

Experience with antivirals during a pandemic and in patients with avian influenza is sparse. Given the timing of these events, there is only limited experience with M2 inhibitors and none with NAIs in pandemic influenza. In some recent cases of avian influenza, NAIs were trialled, either alone or with glucocorticoids and broad-spectrum antibiotics, late in the course of the disease. They do not appear to have reduced mortality, although early initiation of antivirals was reported to be beneficial in a small subset of patients in which cultivable virus disappeared within 2 or 3 days. However, the small number of patients involved and the lack of rigorous study design do not enable accurate assessment of the effectiveness of antivirals in patients with H5N1 influenza [7].

While various factors suggest that a human-human transmissible form of $\mathrm{H} 5 \mathrm{~N} 1$ could be the cause of the next pandemic, it is not known when the next pandemic will occur, whether it will be caused by this strain or another new virus, or how severe it will be.

Certainly, vaccination is the most effective means of preventing influenza. However, a strain-specific vaccine is unlikely to be available during the initial wave of a pandemic. Targeted vaccination (once a vaccine became available) and targeted use of effective antiviral drugs in priority groups has been recommended. Even so, this strategy alone may be too little or too late to decrease mortality, morbidity or the spread of influenza in the general population. Large-scale vaccine production and delivery, all of which are well beyond current global capacity, will be needed to protect against subsequent waves of the pandemic. Broader public health strategies and measures, such as narrow epidemiological surveillance, social distancing, travel limitations and border controls, may also be called for.

The inevitable influenza pandemic looms as an enormous public health threat that challenges decision makers at all levels of healthcare systems. What is the evidence base for meeting this challenge? How do we assemble and present this evidence for different decision makers? Certainly, this evidence arises from no single research method or discipline [8].

\section{VALUE OF THE HEN REPORT}

The principal added value of this HEN synthesis report is that it is based on a transparent review of different types of research studies. This multi-method approach is used in evaluative research to capture the complexity of an issue, without rejecting a priori any particular epidemiological, economic and clinical approach, and without relying upon opinion-based reports. This type of report allows the identification of both pitfalls of available research data and gaps in knowledge to inform decision makers while providing clues about research needs. In addition, this type of report facilitates an understanding of the current healthcare context. The knowledge base for a rapidly evolving issue, such as the threat of pandemic influenza, is subject to sudden obsolescence. New developments unfold almost daily about the global spread of avian influenza, human case experience, mutating influenza strains, and emerging resistance to antiviral drugs.

The barrage of emerging information and news reports can also be inconsistent, contradictory or overwhelming to policy makers. In the instance of pandemic influenza, this may arise from the uncertainty associated with the anticipated levels of mortality and morbidity, and the effectiveness of prevention and treatment strategies. The proportion of patients with laboratory-confirmed influenza in the more selective context of RCTs is likely to be higher than that of patients identified in community practice who truly suffer from influenza. Likewise, the treatment effects of antivirals in community practice are likely to be lower than those observed in the RCTs that are incorporated into the available systematic reviews. Studies with oseltamivir and zanamivir in animal models with recent strains of H5N1 indicate that higher doses and longer regimens of these agents are needed to be effective. The options for using antivirals are limited by the rising resistance of prevailing influenza strains, the possibility that pandemic strains may require higher doses and longer treatment regimens, and the high costs of some antivirals. In community practice prevention, the percentage of risk reduction may vary based on the population strategy employed and the choice of antiviral drug.

These findings have substantial implications for public and private health sectors. At least 50 mostly developed countries are moving to stockpile limited supplies of antiviral drugs. Some are seeking to stockpile limited supplies of experimental vaccine for the H5N1 strain. Of course, the effectiveness of 
stockpiling strategies remains uncertain; a stockpiled vaccine can be of little or no use against the virus that emerges as the pandemic agent, and any effectiveness of antivirals subsides when the course of treatment ends.

Within this uncertain context, physicians must respond to patients' queries knowing that many of the newer vaccines and antiviral interventions recommended for use in an emerging pandemic have been tested only during the last few years in small clinical trials involving healthy patients, and not in people actually exposed to influenza or in the context of an actual epidemic or pandemic. The use of NAIs late on in the course of avian influenza in humans did not appear to reduce mortality, although early initiation of antiviral agents was reported to be beneficial in a small number of patients [7]. The lack of other aspects of robust study design does not enable rigorous assessment of the effectiveness of antivirals in patients with $\mathrm{H} 5 \mathrm{~N} 1$ influenza.

A small set of systematic reviews of the general clinical impact and cost-effectiveness of influenza vaccination and antivirals do exist and are cited in the HEN synthesis report. However, some of the relevant studies consider aspects of certain strategies in a limited, not global, context and rely heavily upon non-systematic reviews or expert consensus. Some of the studies used modelling and related analyses (based in part on clinical or epidemiological evidence) that rely on incomplete evidence or evidence not derived from rigorous studies in research or practice settings.

Recent reports from clinical investigations [9] and a systematic review [10] confirm the appearance of resistance during antiviral treatment of $\mathrm{H} 5 \mathrm{~N} 1$ infection and that the efficacy of antivirals for H5N1 influenza in otherwise healthy adults during the outbreaks in South-East Asia was not associated with any obvious effect on mortality but with a reduction of symptoms.

Medical practice is often a probabilistic exercise, and uncertainties abound in the professional lives of physicians. Nevertheless, transparent multi-method syntheses of research reports can help diminish uncertainty among physicians and other decision makers in healthcare, while supporting more informed decisions for the good of current and future patients.

We face a global public health challenge of potentially great magnitude. Available evidence alone does not point to a single best strategy to confront this great challenge. Nevertheless, objective appraisal of available evidence leads unambiguously to the need for immediate collaborative strategies involving: public health institutions and the private sector; physicians, public health professionals and researchers; and wealthy and less-developed countries. The inevitable pandemic influenza presents a scenario of increasingly complex healthcare issues and circumstances that require cross-sectorial and global approaches.

\section{REFERENCES}

1 Taubenberger JK, Reid AH, Lourens RM, et al. Characterization of the 1918 influenza virus polymerase genes. Nature 2005; 437: 889-893.

2 Langley JM, Faughn ME. Prevention of influenza in the general population. CMAJ 2004; 171: 1213-1222.

3 Jefferson T, Rivetti D, Rivetti A, et al. Efficacy and effectiveness of influenza vaccines in elderly people: a systematic review. Lancet 2005; 366: 1165-1174.

4 Centers for Disease Control and Prevention. High levels of adamantine resistance among influenza A (H3N2) viruses and interim guidelines for use of antiviral agents - United States, 2005-06 influenza season. MMWR 2006; 55: 44-46.

5 WHO Guidelines on the Use of Vaccines and Antivirals During Influenza Pandemics. Geneva, World Health Organization, 2004. www.who.int/csr/resources/publications/influenza/11_29_01_A.pdf. Date last accessed: November 17, 2005.

6 Kaiser L, Wat C, Mills T, et al. Impact of oseltamivir treatment on influenza-related lower respiratory tract complications and hospitalizations. Arch Intern Med 2003; 163: $1667-1672$.

7 Beigel JH, Farrar J, Han AM, et al. Avian influenza A (H5N1) infection in humans. N Engl J Med 2005; 353: 1374-1385.

8 Granados A. Health technology assessment and clinical decision-making: which is the best evidence? Int J Technol Assess Health Care 1999; 15: 585-614.

9 de Jong MD, Tran TT, Truong HK, et al. Oseltamivir resistance during treatment of influenza (H5N1) infection. N Engl J Med 2005; 353: 2667-2672.

10 Jefferson T, Demicheli V, Rivetti D, et al. Antivirals for influenza in healthy adults: systematic review. Lancet 2006; 367: 303-313. 